Supporting Information for

\title{
Spatial Confinement as an Effective Strategy for Improving the Catalytic Selectivity in Acetylene Hydrogenation
}

\author{
Qiang Fu, ${ }^{*}+$, Fan $\mathrm{Wu},{ }^{\dagger}$ Bingxue Wang, ${ }^{\dagger}$ Yuxiang $\mathrm{Bu},{ }^{\dagger}$ and Claudia Drax ${ }^{\ddagger}, \uparrow$
}

$\dagger$ School of Chemistry and Chemical Engineering, Shandong University, Jinan 250100, China

$\$$ Institut für Physik and IRIS Adlershof, Humboldt-Universität zu Berlin, Berlin 12489, Germany \Fritz-Haber-Institut der Max-Planck-Gesellschaft, Berlin 14195, Germany

*Email: qfu@sdu.edu.cn 

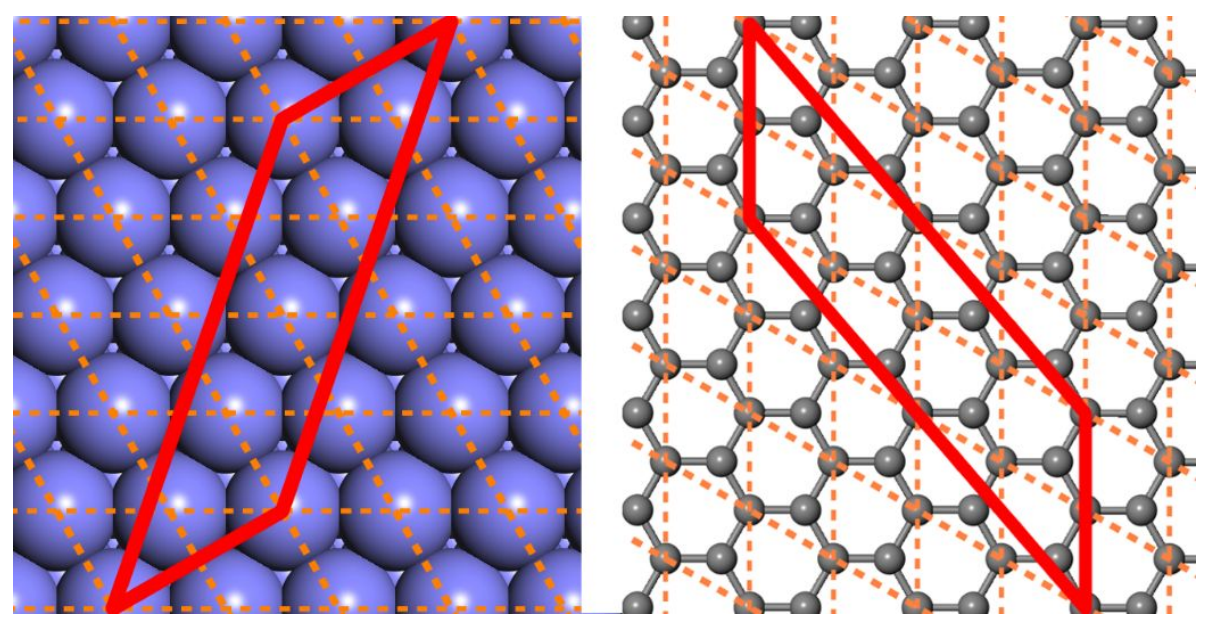

Figure S1: Lattices of the $\operatorname{Ag}(111)$ surface and the graphene monolayer in the construction of the interface model. The corresponding unit cells indicated by the red solid lines. The slate blue and gray spheres represent Ag and C atoms, respectively. The original unit cell of each component is marked by orange dashed lines.

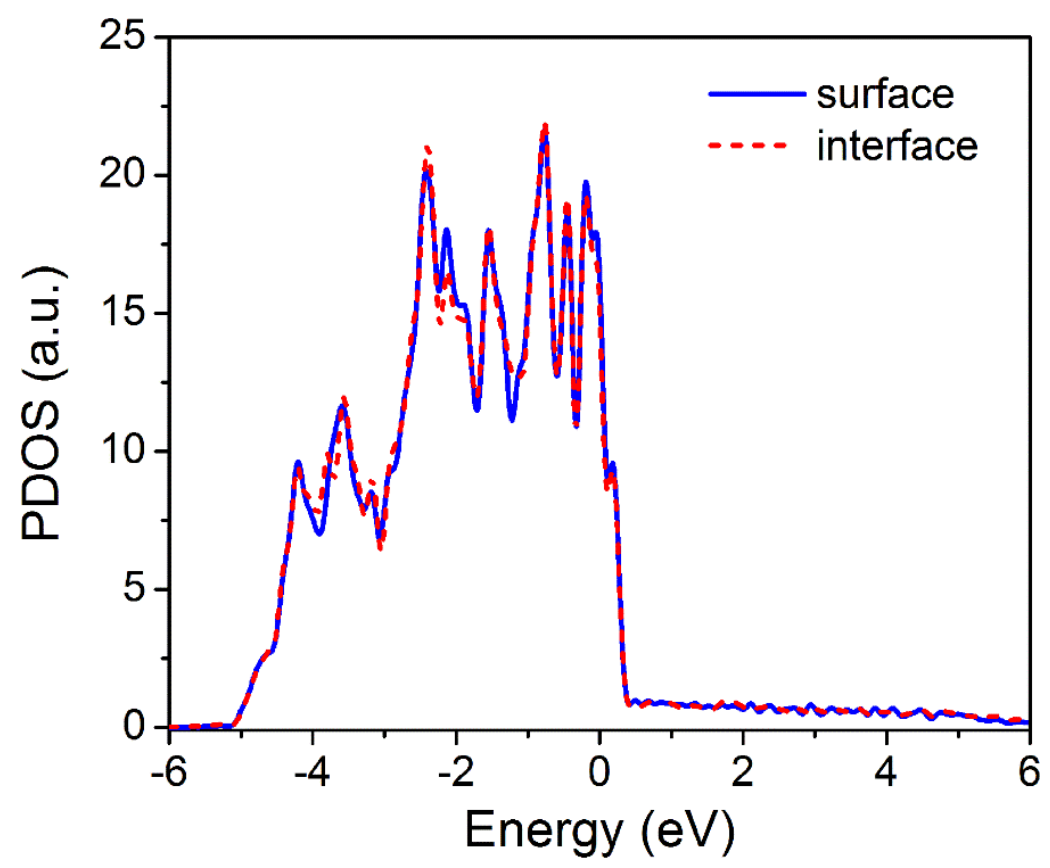

Figure S2: Density of states (DOS) of the $d$ electrons of $\operatorname{Pd}(111)$ before (blue solid line) and after (red dashed line) the coverage of the graphene overlayer. 

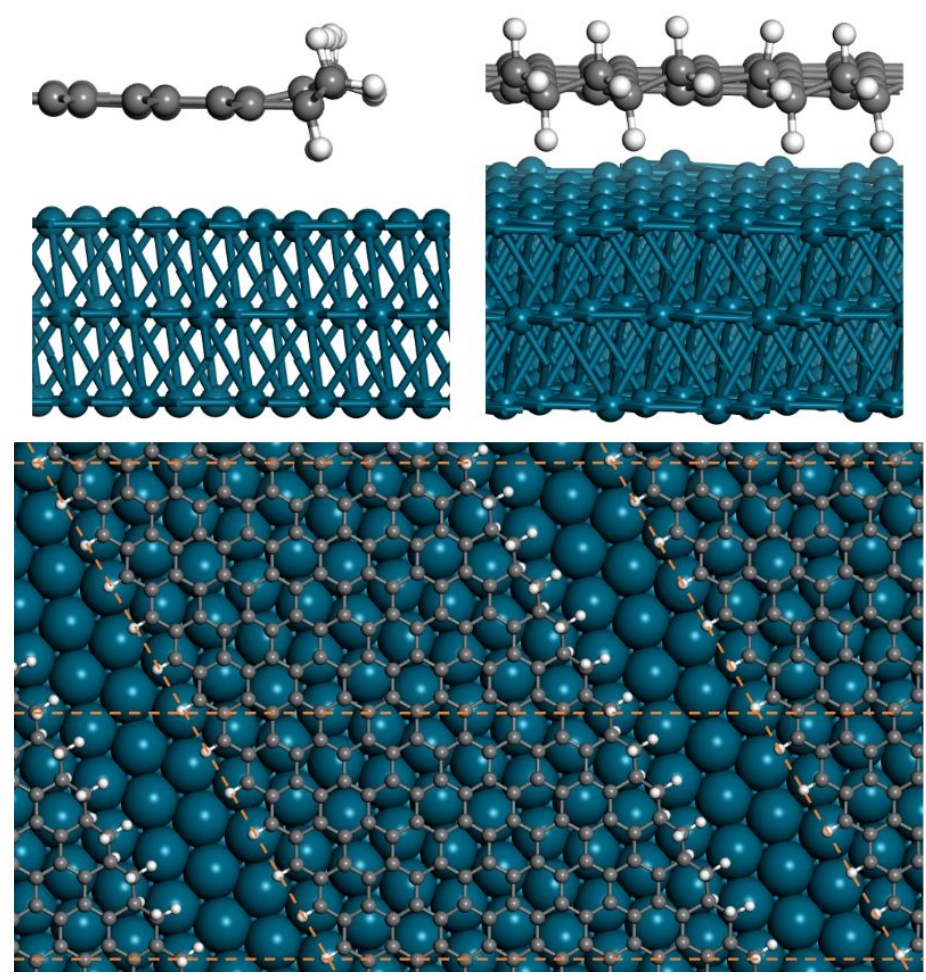

Figure S3: Simulation model of the inlet region for the acetylene intercalation into the subnano space between graphene and the $\mathrm{Pd}(111)$ substrate. The teal blue, gray, and white spheres represent $\mathrm{Pd}, \mathrm{C}$, and $\mathrm{H}$ atoms, respectively. The lattice of the supercell is marked by orange dashed lines. 

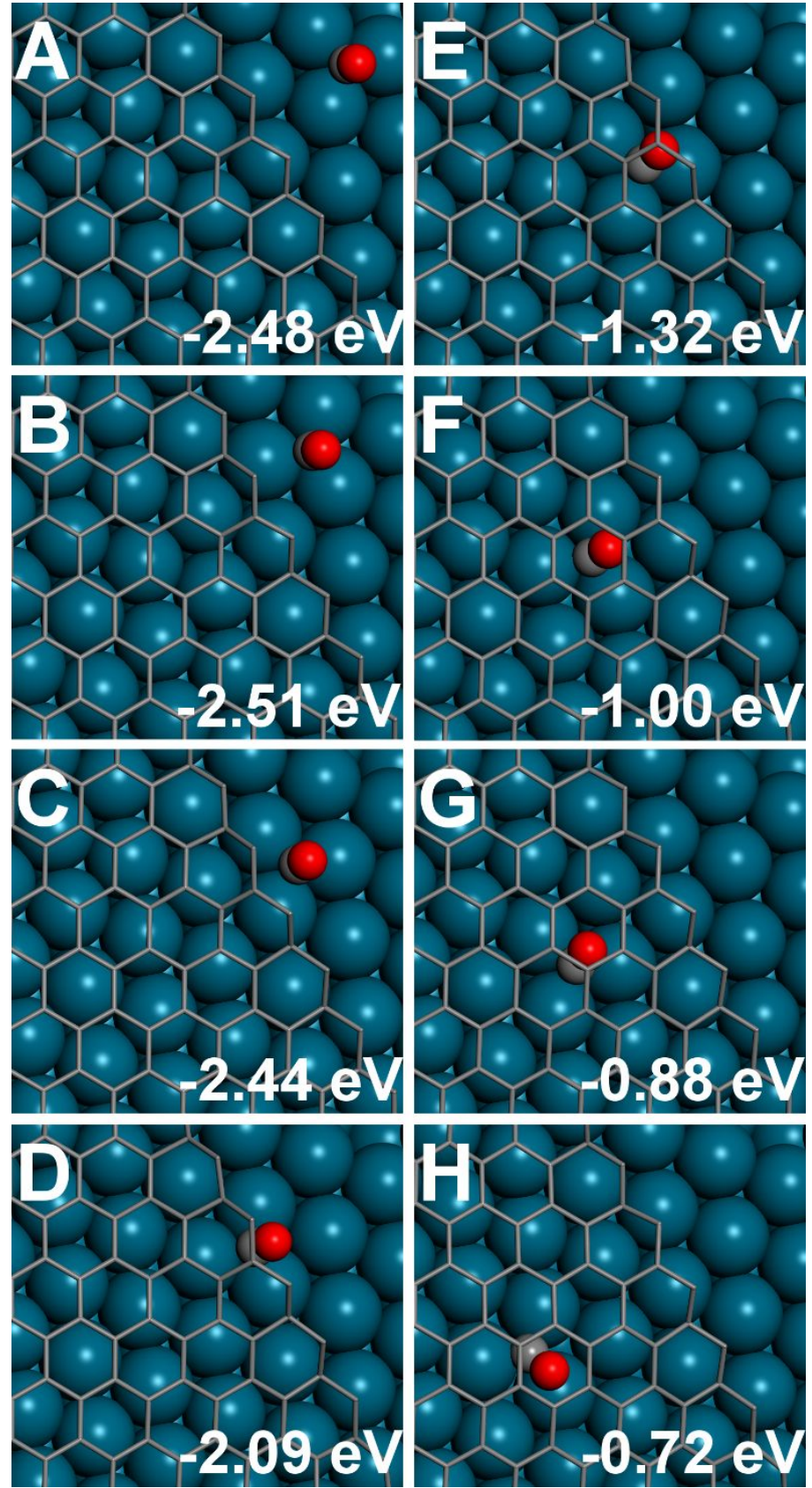

Figure S4: Selected intermediate states (A-H) in the entire process of a $\mathrm{CO}$ molecule entering the subnano space formed by graphene and $\mathrm{Pd}(111)$. The adsorption energies of $\mathrm{CO}$ are labeled in the lower right corners of the respective figures. The hydrogen atoms used to saturate the edge carbon atoms are not shown for the sake of clarity. 